\title{
What is the impact of a previously failed Robicsek repair in the subsequent treatment of sternal dehiscence with thermoreactive nitinol clips?
}

\author{
Yunus Seyrek $^{1}$ and Murat Akkuş ${ }^{1}$ \\ ${ }^{1}$ Istanbul Mehmet Akif Ersoy Training and Research Hospital
}

April 7, 2021

\begin{abstract}
Background: In this study, we conducted a retrospective review of patients at our institution with noninfectious sternal dehiscence (NISD) after median sternotomy who received thermoreactive nitinol clips (TRNC) treatment during a 10-year period. We compared TRNC patients with and without history of failed Robicsek repair. The purpose of the study was to analyze the impact of previous Robicsek repair on the treatment of sternal dehiscence with TRCN. Methods: Between December 2009 and January 2020, out of 283 patients with NISD who underwent refixation, we studied 34 cases who received TRNC treatment. We divided these 34 cases into two groups: patients who had a previously failed Robicsek procedure before TRNC treatment (group $\mathrm{A}, \mathrm{n}=11$ ) and patients who had been directly referred to TRCN treatment (group $\mathrm{B}, \mathrm{n}=23$ ). Results: Postoperative complication rate was significantly higher in group A $(\mathrm{p}=0.026)$. Hospitalization duration was significantly longer in group A due to the higher complication rate $(\mathrm{p}=0.001)$. Operative time was significantly shorter and blood loss was significantly lower in group B ( $\mathrm{p}=0.001)$. Conclusion: The Robicsek procedure is considered an effective method in the treatment of NISD but, in case of its failure, subsequent TRNC treatment might become cumbersome in high-risk patients. In our study, a previously failed Robicsek procedure caused significantly higher morbidity and additional operative risk in later TRNC treatment of high-risk cases. Ultimately, we speculate that a direct TRNC treatment for NISD is favorable in high-risk patients.
\end{abstract}

\section{Introduction:}

Full median sternotomy is the preferred surgical approach for exposing the heart in cardiac surgery. The current standard method for sternotomy closure is wire circlage, usually referred to as the conventional technique [1]. Most surgical studies report that early sternotomy wound dehiscence occurs in heart surgery with an incidence from $0.5-10 \%$ [2]. Sternal wound dehiscence is defined as the instability and separation of both sternal halves with or without an open skin wound infection.

There are many methods for reconstruction of median sternotomy wound dehiscence in cardiac surgery, although the Robicsek technique is the most common [3]. The thermoreactive nitinol clips (TRNC) system is reported to be quite useful; however, it is not well established whether it can be used routinely instead of established methods, such as the Robicsek technique [4].

In this study, we conducted a retrospective review of patients at our institution with noninfectious sternal dehiscence (NISD) after median sternotomy who received TRNC treatment during a 10-year period. We compared TRNC patients with and without history of failed Robicsek repair. Our aim was to analyze the impact of previous Robicsek repair on the treatment of sternal dehiscence with TRCN.

\section{Material and Methods:}

This retrospective study was approved by the local ethics committee and have been performed in accordance with the ethical standards laid down in Declaration of Helsinki. Between December 2009 and January 2020, a 
total of 21,357 median sternotomies were performed, with an incidence of NISD of $1.32 \%(283 / 21,357)$. The incidence of infectious sternal dehiscence during the same period was $2.1 \%(432 / 21,357)$. Sternal instability was determined at hospital and was defined as the presence of mobility when pressing both sternal halves separately. Treatment failure was defined as the need for reintervention for sternal closure.

Institutional protective measures against major wound infection and sternal dehiscence included: a bath with chlorhexidine soap the night before the operation, surgical site antisepsis with $10 \%$ povidone-iodine solution, whole body shaving two days before the operation, antibiotic prophylaxis with $1 \mathrm{~g}$ cefazolin $(2 \mathrm{~g}$ in patients $>80 \mathrm{~kg}$ ), and use of a chest brace and activity restriction for 6 to 8 weeks after the surgery. Bone wax was not used during the procedures. Sternal closure included the placement of 6-8 stainless steel wire U-sutures one-by-one, without crossing.

We considered three principal categories for the classification of sternal wound complications: mechanical NISD, sternal dehiscence with superficial wound sternal infection, and sternal dehiscence with deep sternal wound infection [5]. Patients who experienced infectious complications of the sternal wound after sternotomy $(\mathrm{n}=432)$ were excluded from the study. Of 283 patients with NISD who underwent refixation, we mainly studied 34 cases who received TRNC treatment. We divided these 34 cases into two groups: patients who had a previous Robicsek repair before TRNC treatment (group $\mathrm{A}, \mathrm{n}=11$ ) and patients who had been directly referred to TRCN treatment (group $\mathrm{B}, \mathrm{n}=23$ ). In addition, we compared the results of those who underwent TRCN treatment (group B, $\mathrm{n}=23$ ) with all 260 patients who underwent Robicsek repair (group C, $\mathrm{n}=260$ ) as the first sternal refixation procedure (Figure1). Demographic, preoperative, intraoperative and postoperative parameters were analyzed.

Nitinol thermoreactive clips (IAWA Bioscience Engineering Company, Jiangsu, China) were used. Operation time was defined as the time interval between the introduction of anesthesia and the end of the surgical procedure. Routine preoperative echocardiography examinations were performed. All patients were cultured for infection at the time of reoperation and received standard antibiotic prophylaxis, which included 24-hour administration of a first-generation cephalosporin. Before repair treatment, all cultures were verified to be negative for infective microorganisms. Computerized data of the patients were reviewed for reassessment of those in whom refixation was performed.

High-risk patients were defined as those having three or more risk factors, including comorbidities and surgical complications which predisposed them to sternal dehiscence. All reported comorbidities were established by attending physicians.

Comorbidities included: severe or morbid obesity (body mass index $>35 \mathrm{~kg} / \mathrm{m} 2$ ), old age ( $>75$ years), chronic obstructive pulmonary disease (COPD) (forced expiratory volume less than $70 \%$ in 1 second, and/or need for bronchodilatatory therapy for more than 6 months), congestive heart failure (New York Heart Association functional classes III-IV), peripheral vascular disease (Fontaine classification stages IIB-IV), chest irradiation, diabetes (insulin dependent or non-insulin dependent therapy longer than 12 months), severe osteoporosis (therapy for more than 6 months), chronic steroid use (therapy more than 6 months), renal insufficiency (creatinine $>2 \mathrm{mg} / \mathrm{dL}$ ), and prolonged ventilation (more than 7 days) [6].

Surgical complications included: use of bilateral internal mammary artery grafts, cardio-pulmonary bypass run longer than 5 hours, an asymmetric off-center sternotomy, transverse fractures of the sternum, rewiring for post-operative bleeding, and repeated sternal reopening [7].

Out of 283 patients with NISD; 260 underwent Robicsek repair, of which 17 were reported as high-risk patients. Of these 17 high-risk patients, 11 still had sternal instability after Robicsek treatment. There was no reported failure in the remaining Robicsek cases. Failed cases were referred to the thoracic surgery clinic for TRNC treatment.

The Robicsek method consists of placing continuous wire sutures parasternally. First, substernal tissues or adhesions were dissected. Each half of the sternum was fastened by continuous parasternal wire sutures. The suture began at the upper end of the sternum and passed down to the xiphoid process, alternating anteriorly 
and posteriorly between the costal cartilages. The suturing was then reversed toward the cranial direction, passing through the cartilages posteriorly where it had been anterior, and vice versa. At the upper end of the first rib, adequate reinforcement was provided and the two ends of the suture were tied together. Stainless steel wires were placed parasternally. The first wire was then passed through the manubrium through the second intercostal space and twisted to form a ring. The second wire was placed through the second and third intercostal spaces in an interlocking fashion until the end of the sternum was reached. The interlocking created a chain on both sides of the sternum [8]. The Robicsek technique was performed in the cardiovascular surgery clinic.

The TRNC method includes placement of nitinol clips either with or without the dissection of the substernal tissues. The pectoral muscle was completely bilaterally mobilized from the thoracic wall to facilitate approximation. Granulation tissues at both sternal ends were carefully removed and the bone tissue was exposed. Using electrocautery, parasternal holes were opened through the intercostal muscles close to the parasternal edges. When dissection of the substernal tissues or adhesions was required, they were dissected close to the costochondral junction using a right angle instrument. Both hemi-sternums were joined and stabilized with two Backhaus forceps. The sternal horizontal diameters were measured with the sizing tool provided, and appropriately-sized nitinol clips were used. The clips were put into refrigerated $\left(<5^{\circ} \mathrm{C}\right)$ sterile saline solution. They became forgeable in minutes and were adequately enlarged by bending. The clips were inserted into the parasternal holes, from superior to inferior direction, and at least four horizontal placements were made for each patient. The clips were then heated with warm water $\left(>45^{\circ} \mathrm{C}\right)$. After the clips resumed their original shapes and regained stiffness, the forceps were removed. The TRNCs were placed at the intercostal spaces without using steel wires. Pectoral muscle flaps were closed at the midline using absorbable sutures. Small suction drains were left in the central defect and under both pectoral muscle flaps and the substernum. The TRNC technique was performed at the thoracic surgery clinic (Figure 2). In both reoperations, mediastinal tissue and fluid cultures were collected in patients with suspected infections.

All statistical analyses were performed using SPSS version 21.0 software (SPSS, Inc., Chicago, IL, USA). Continuous parameters are presented as mean standard deviations. Categorical data are presented as numbers and percentages, and proportions between the two groups were compared using the chi-square test or Fisher's exact test. The Mann-Whitney U test was used for comparison of nonparametric variables, and the independent samples t test was used for comparison of parametric variables. A p value $<0.05$ was considered significant.

\section{Results:}

The retrospective cohort included 283 patients who underwent refixation due to NSID after median sternotomy. The patients had a mean age of 58.5 \pm 13.4 years (range, 33-82 years) at reoperation and a male $(\mathrm{n}=176)$ to female $(\mathrm{n}=107)$ ratio of 1.64 . The mean interval between the initial operation and the sternal reoperation was $49.4 \pm 9.5$ days (range, $42-78$ days).

Our study mainly consisted of 34 patients who were treated with the TRNC method due to NISD after median sternotomy. Demographic, preoperative, intraoperative, and postoperative variables of patients who had a previous failed Robicsek procedure before the TRNC method (group A, n=11), and patients who underwent the TRNC method immediately (group $\mathrm{B}, \mathrm{n}=23$ ), are presented in Table 1 . The mean interval between failed Robicsek procedures and TRNC treatment was 18.4 \pm 2.6 days (range, 14-26 days) in group A $(n=11)$.

Initial procedures included: $58.8 \%$ coronary artery bypass grafting (20/34), $11.7 \%$ valve replacement (4/34), $5.8 \%$ repair of ascending aorta $(2 / 34)$, and $23.5 \%$ combination of procedures $(8 / 34)$. There was no significant difference in terms of initial procedure requiring median sternotomy between the analyzed groups.

The mean hospitalization time after TRNC treatment was 9.3 \pm 3.2 days (range, 5-24 days). The mean operative time was $56.4 \pm 8.4$ minutes (range, 40-95 minutes) and the mean blood loss was $312.4 \pm 76.6 \mathrm{ml}$ (range, $150-1000 \mathrm{ml}$ ). 
There was no significant difference between the groups in terms of comorbidity rates or surgical complications. Risk distribution according to group A and B, including comorbidities and surgical complications, is presented in Table 2. Both groups consisted of high-risk patients.

Postoperative complications included: chronic ventilator dependence or intubation for more than 7 days $(2.9 \%, 1 / 34)$, pneumonia $(5.8 \%, 2 / 34)$, hematoma $(8.8 \%, 3 / 34)$, seroma $(14.7 \%, 5 / 34)$, pleural effusion $(8.8 \%$, $3 / 34)$, delirium $(11.8 \%, 4 / 34)$, atrial fibrillation $(8.8 \%, 3 / 34)$, and severe limitations of physical activity due to excess sternal pain $(26.4 \%, 9 / 34)$. Postoperative superficial sternal infection was seen in $5.8 \%$ of TRNC patients $(2 / 34)$, but none of the superficial infections progressed into the mediastinum. No mortality was reported. A detailed comparison of post-operative complications between the analyzed groups is presented in Table 3. Postoperative complication rate was significantly higher in group $\mathrm{A}(\mathrm{p}=0.026)$. Hospitalization duration was significantly longer in group $A$, due to the higher complication rate $(\mathrm{p}=0.001)$. Operative time was significantly shorter and blood loss was significantly lower in group B $(\mathrm{p}=0.001)$.

After TRNC treatment, sternal stability at hospital discharge was reached in $94.1 \%$ of the patients $(32 / 34)$. Two cases in group A still had sternal dehiscence (2/11), whereas all patients in group B had sternal stability at the time of discharge $(\mathrm{p}=0.035)$.

In addition, we compared the results of group B and group C. High-risk patient frequency was significantly higher in TRCN group (group B) compared to Robicsek group (group C) (100\% vs 6.5\%) ( $\mathrm{p}=0.001)$. Overall success rate of Robicsek repair was $93.8 \%$ (244/260) and TRCN was 100\% ( $\mathrm{p}>0.05)$ but the success rate of the Robicsek repair in high-risk patients with NISD ( $\mathrm{n}=17)$ was $35.3 \%(6 / 17)$. TRCN treatment was significantly more effective in high-risk NISD patients compared to Robicsek repair with a success rate of $100 \%$ ( $\mathrm{p}=0.01)$. Lastly, operative time was significantly shorter in group B compared to group C (47.3 \pm 7.6 vs $75.3 \pm 4.8)(\mathrm{p}=0.01)$. There was no significant difference between the groups in terms of age, gender, blood loss, complication rate, ICU stay or hospital stay $(\mathrm{p}>0.05)$.

\section{Discussion:}

Sternal dehiscence represents one of the major causes of morbidity after cardiac surgery performed through full median sternotomy. The key to the successful management of NISD is early referral to refixation [9]. Time to reoperation for refixation varies in the literature from 10 to 300 days $[4,6]$. In our study, refixation was performed after a mean time of $49.4 \pm 9.5$ days following the initial procedure. Surgery for this complication should be performed as soon as possible.

Multiple surgical techniques have been described for reconstructing the anterior chest wall in the treatment of sternal dehiscence, but none of them is yet considered the gold-standard procedure [10]. In our hospital, Robicsek repair is the first method of treatment in NSID after median sternotomy whereas TRNC method is mostly advised for patients with multiple comorbidities. In this study, we compared the surgical results of the TRNC technique in patients with a previously failed Robicsek treatment (group A) and patients who were directly referred for TRNC treatment after a diagnosis of NISD (group B). Moreover, we compared group $\mathrm{B}$ with those who underwent Robicsek repair as the first procedure (group C). Our aim was to establish the importance of prioritization of TRNC treatment in high-risk NISD patients.

The potential mechanisms of comorbidities that increase the risk of sternal dehiscence in high-risk patients are as follows: the sternum healing is compromised due to the separating radial forces from the prolonged ventilation and chronic abundant cough in COPD; the sternum bone fragility is induced by the intrinsic pathology in patients $>75$ years of age, diabetes and severe osteoporosis; and that osteosynthesis is reduced in patients with renal insufficiency, chest irradiation and chronic steroid use. Additional risk factors could include congestive heart failure and peripheral vascular disease, because these patients have particularly compromised peripheral vascularization, which reduces sternal healing [11]. In morbidly obese patients, the lateral stress is increased even more because of their body habitus. Excessive tissue places additional stress on the sternotomy closure, both laterally by the chest wall and inferiorly by the abdominal wall [12]. In our study, there was no significant difference between the groups in terms of comorbidities. 
Bilateral internal mammary artery usage interrupts sternal blood flow, which poses a risk for sternal dehiscence. Prolonged retraction of both sternum halves may cause ischemic fields and result in healing problems after long cardio-pulmonary bypass runs, repeated sternal openings, and rewirings. An asymmetric off-center sternotomy and transverse fractures prevent a decent anatomical sternal closure due to disruption of sternochondral joints and sternum halves (Figure 3) [13]. In our study, there was no significant difference between the groups in terms of surgical complications. Both group A and group B consisted of high-risk patients.

The classic technique described by Robicsek et al. relocates and distributes the pressure over the sternum by changing the site of the pressure and providing wider support [8]. The disadvantage of this technique is the need for substernal dissection and the effect on the blood flow to the area. When intercostal arteries are squeezed by the ring formed by steel wires running up-and-down and anterior-posterior around the ribs, a constrictive weave is produced that can disrupt the collateral blood supply to the sternum. This may worsen a pre-existing ischemia, which facilitates bacterial colonization and delays sternal healing. Furthermore, ischemia may cause bone necrosis and additional sternal fragmentation [13,14]. In our study, 260 patients underwent Robicsek repair due to NISD, but $6.1 \%$ had still sternal dehiscence afterward $(\mathrm{n}=16)$. Out of those 16 patients, 11 cases were high-risk patients and they were referred to TRNC treatment. The success rate of the Robicsek method in the treatment of high risk NISD patients, was $35.3 \%$ (6/17). On the other hand, its fail rate was only $2.1 \%$ in without high-risk patients $(5 / 243)$.

The TRNC technique is easier and has a shorter operation time than the Robicsek method. In our study, operative time in group B was significantly shorter compared to group C as expected. The TRNC method is also considered a safer technique because less substernal dissection is needed. TRNCs have shown less risk for tearing the bone than steel wires. Nitinol clips are not integrated into the bone and their thermoreactive characteristics allow them to be removed easily when required [15]. The TRNC technique brings both hemisternums together without harming the intercostal structures. Therefore, it does not technically affect sternal blood flow [16]. Many studies have hypothesized that using TRNCs is a superior dehiscence repair method compared to Robicsek method $[8,17]$. The disadvantage of the TRNC technique is that it is more expensive due to the cost of hardware. The cost of each plate ranges from 90 euro to 100 euro. In our study, we only used TRNCs in high-risk patients and patients with failed Robicsek procedures. We wished to use the TRNC method in each patient with NISD, but its cost prevented us from performing this method on regular basis. After observing several cases of Robicsek repair failure in high-risk patients, most high-risk patients with NISD were directly referred to the thoracic surgery clinic for TRNC treatment.

In our study, we observed that ischemic tissues were more common, the sternum often adhered to the underlying tissue, and sometimes to the myocardium, due to previous Robicsek procedure during TRNC treatment in group A. Since group B had no history of failed a Robicsek method, the surgeon could perform a rapid and less challenging TRNC treatment without additional surgical risks, due to significantly less need for substernal dissection. In our study, we did not perform substernal dissection in group B. Consequently, operation time and operative blood loss in group B was significantly lower compared to group A. Moreover, nonunion rate was significantly lower in group B.

According to various studies, the most commonly reported complications of TRNC treatment are: postoperative pneumonia with an incidence up to $10 \%$, hematoma or seroma formation with an incidence up to $24 \%$, and clip removal due to infection or pain up to $50 \%$ [18]. Current sternal dehiscence treatment methods have morbidity rates of $10-25 \%$ and mortality rates of $5-47 \%$ [19]. Refixation has a high risk of postoperative complications regardless of the chosen treatment method [20]. In our study, although both group A and B consisted of high-risk patients, the postoperative complication rate in group A was significantly higher than in group B (54.5\% vs $17.4 \%$ ). Due to this higher complication rate, hospitalization in group A was also significantly longer compared to that in group B. Postoperative wound complication and pneumonia rates were significantly higher in group A. As a result, an additional operation for refixation and the introduction of an extra session of general anesthesia for patients with additional diseases greatly increased both surgical risks and postoperative complication rates. Fortunately, there was no mortality in either group.

Our study was a single center, retrospective study. We did not include patients with infectious sternal 
dehiscence due to lack of recorded data. Therefore, we could not come to a general conclusion about the usage of the TRNC system in high-risk patients and were limited to NISD. Due to the small number of patients included in our study, statistical power was low and our ability to examine low-frequency outcomes was limited.

Patients with multiple established factors of sternal dehiscence are high-risk patients for the treatment of NISD. TRNC use in sternal closure of high-risk patients may prevent sternal dehiscence, but it is impractical to use such expensive materials on regular basis. The Robicsek procedure is proven to be an effective method in the treatment of NISD but, in case of its failure, subsequent TRNC treatment might become cumbersome in high-risk patients. In our study; a direct TRNC treatment approach for high-risk patients in the treatment of NISD was superior to the Robicsek method because a previously failed Robicsek procedure caused significantly higher morbidity and additional operative risk in later TRNC treatment of high-risk cases. Ultimately, we speculate that a direct TRNC treatment for NISD is favorable in high-risk patients.

Acknowledgements : English editing by 'Editage'

Conflict of interest : none

Funding: None

\section{References:}

1. Douville EC, Asaph JW, Dworkin RJ, et al. Sternal preservation: A better way to treat most sternal wound complications after cardiac surgery. Ann Thorac Surg 2004;78:1659-64.

2. Hosseinrezaei H, Rafiei H, Amiri M. Incidence and risk factors of sternal wound infection at site of incision after open-heart surgery. J Wound Care 2012;21:408-11.

3. Dell'Amore A, Campisi A, Giunta D, Congiu S, Dolci G, Murana G, Suarez SM, Daddi N. Surgical options to treat massive sternal defect after failed Robicsek procedure. Journal of thoracic disease. 2018 Jun;10(6):E410.

4. Sarıkaya S, Aksoy E, Özen Y, Dedemoğlu M, Özgür MM, Büyükbayrak F, Kırali K. Thermoreactive nitinol clips: propensity score comparison with Robicsek technique. Asian Cardiovascular and Thoracic Annals. 2015 May;23(4):399-405.

5. Schimmer C, Reents W, Berneder S, et al. Prevention of sternal dehiscence and infection in high-risk patients: A prospective randomized multicenter trial. Ann Thorac Surg 2008;86:1897-904.

6. Olbrecht VA, Barreiro CJ, Bonde PN, Williams JA, Baumgartner WA, Gott VL, Conte JV. Clinical outcomes of noninfectious sternal dehiscence after median sternotomy. The Annals of thoracic surgery. 2006 Sep 1;82(3):902-7.

7. Song DH, Lohman RF, Renucci JD, Jeevanandam V, Raman J. Primary sternal plating in high-risk patients prevents mediastinitis. European journal of cardio-thoracic surgery. 2004 Aug 1;26(2):367-72.

8. Robicsek F, Daugherty HK, Cook JW. The prevention and treatment of sternum separation following open-heart surgery. The Journal of thoracic and cardiovascular surgery. 1977 Feb 1;73(2):267-8.

9. Wong CH, Senewiratne S, Garlick B, Mullany D. Two-stage management of sternal wound infection using bilateral pectoralis major advancement flap. European journal of cardio-thoracic surgery. 2006 Jul 1;30(1):148-52.

10. Sanna S, Brandolini J, Pardolesi A, et al. Materials and techniques in chest wall reconstruction: a review. J Vis Surg 2017;3:95.

11. Bejko J, Bottio T, Tarzia V, De Franceschi M, Bianco R, Gallo M, Castoro M, Bortolussi G, Gerosa G. Nitinol flexigrip sternal closure system and standard sternal steel wiring: insight from a matched comparative analysis. Journal of Cardiovascular Medicine. 2015 Feb 1;16(2):134-8.

12. Liao JM, Chan P, Cornwell L, Tsai PI, Joo JH, Bakaeen FG, Luketich JD, Chu D. Feasibility of primary sternal plating for morbidly obese patients after cardiac surgery. Journal of Cardiothoracic Surgery. 2019 Dec;14(1):1-7.

13. Robicsek F, Fokin A, Cook J and Bhatia D. Sternal instability after midline sternotomy. Thorac Cardiovasc Surg 2000; 48: 1-8. 
14. Sharma R, Puri D, Panigrahi BP and Virdi IS. A modified parasternal wire technique for prevention and treatment of sternal dehiscence. Ann Thorac Surg 2004; 77: 210-213.

15. Centofanti P, La Torre M, Barbato L, Verzini A, Patane'F and di Summa M. Sternal closure using semirigid fixation with thermoreactive clips. Ann Thorac Surg 2002;74: 943-945.

16. Sahasrabudhe P, Jagtap R, Waykole P, Panse N, Bhargava P, Patwardhan S. Our experience with pectoralis major flap for management of sternal dehiscence: A review of 25 cases. Indian journal of plastic surgery: official publication of the Association of Plastic Surgeons of India. 2011 Sep;44(3):405.

17. Pai S, Gunja NJ, Dupak EL, McMahon NL, Roth TP, Lalikos JF, Dunn RM, Francalancia N, Pins GD, Billiar KL. In vitro comparison of wire and plate fixation for midline sternotomies. The Annals of thoracic surgery. 2005 Sep 1;80(3):962-8.

18. Plass A, Emmert MY, Pilsl M, et al. Sternal plate closure: indications, surgical procedure and followup. Thorac Cardiovasc Surg 2011;59(1):30-33

19. Landes G, Harris PG, Sampalis JS, Brutus JP, Cordoba C, Ciaburro H, Bernier C, Nikolis A. Outcomes in the management of sternal dehiscence by plastic surgery: a ten-year review in one university center. Annals of plastic surgery. 2007 Dec 1;59(6):659-66.

20. Vos RJ, Jongbloed L, Sonker U, Kloppenburg GT. Titanium plate fixation versus conventional closure for sternal dehiscence after cardiac surgery. The Thoracic and cardiovascular surgeon. 2017 Jun;65(04):338-42.

\section{Figure Legends:}

Figure 1. Study flowchart

Figure 2. Phases of Thermoreactive Nitinol Clips Treatment

Figure 3. Visual of transverse sternal fractures after asymmetric off-center sternotomy

Table 1. Demographic, preoperative, intraoperative, and postoperative variables: Comparison between group A and group BTable 2. Comparison of risk factors including comorbidities and surgical complications between group A and group BTable 3. Comparison of post-operative complications between group A and group B

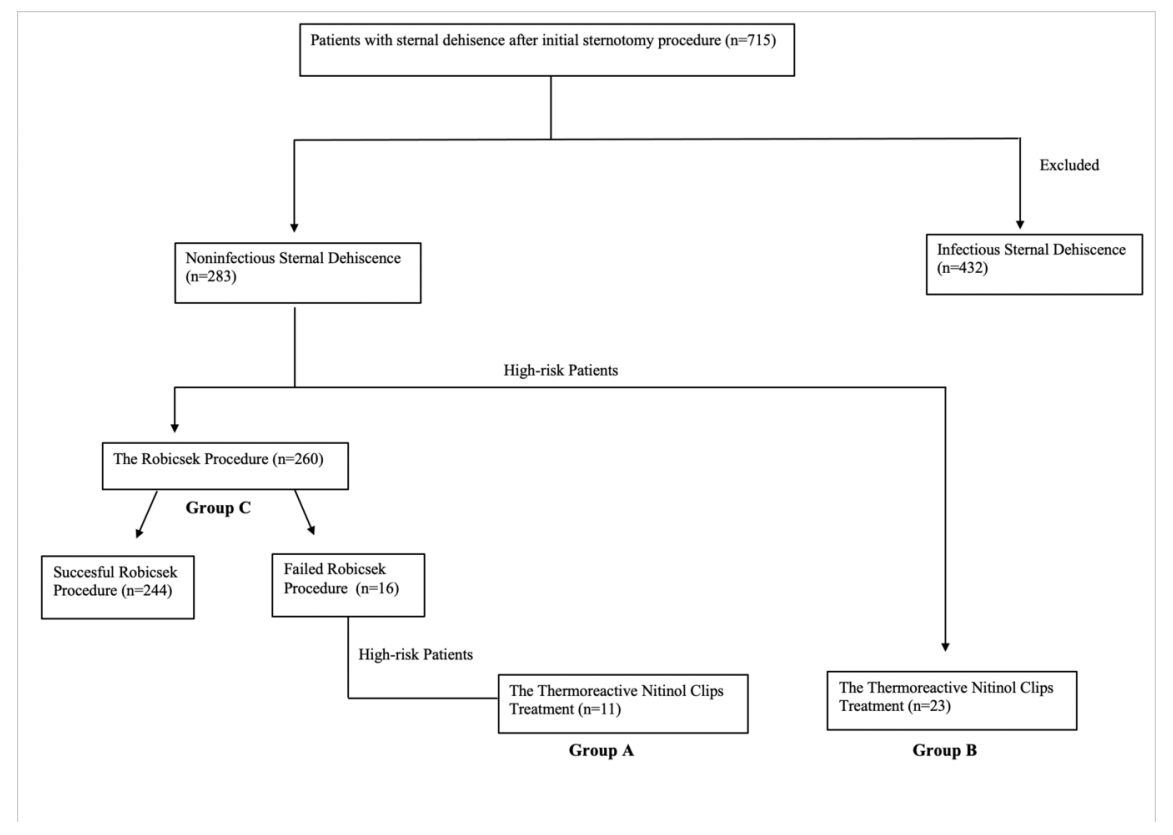



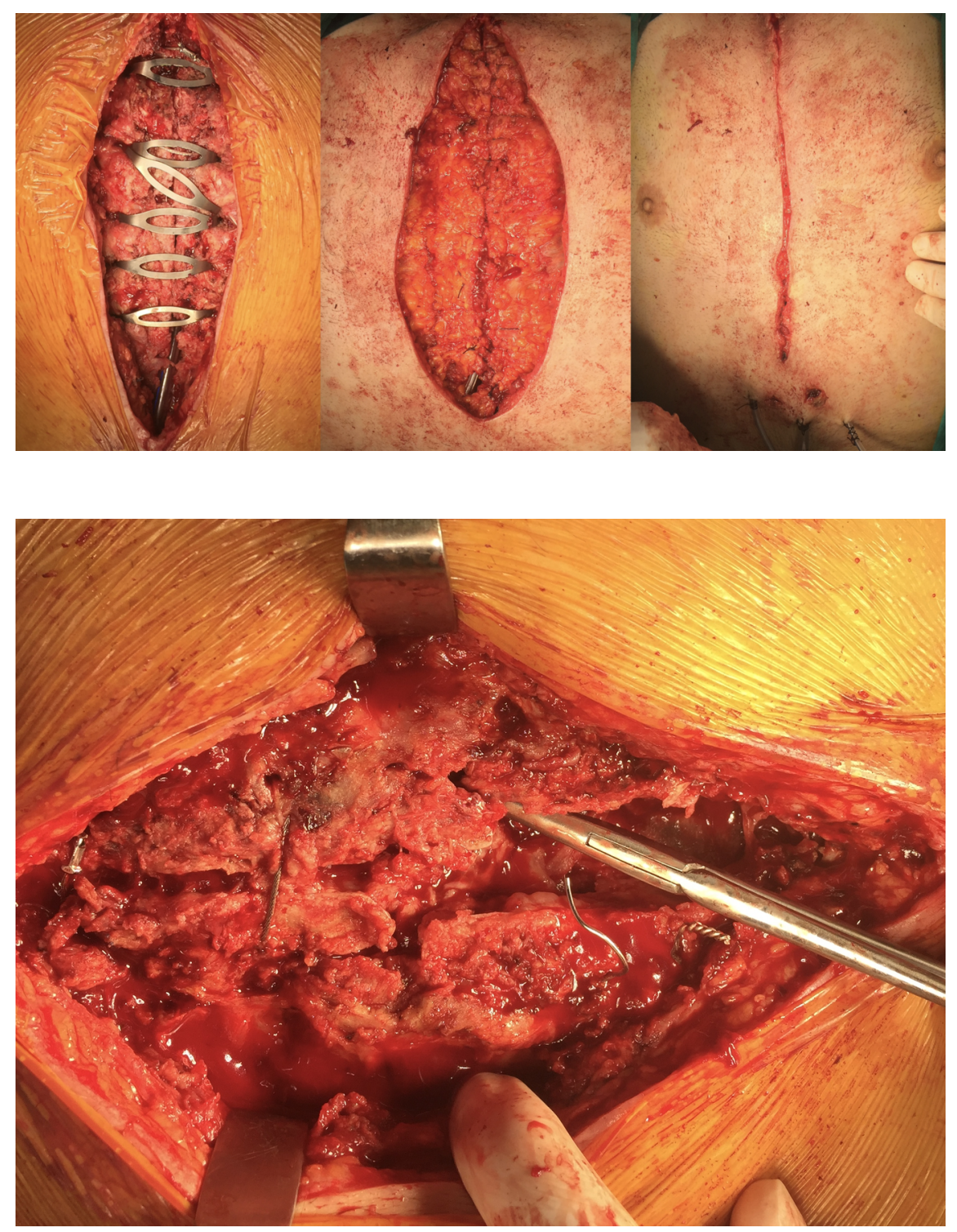

\section{Hosted file}

Table 1.pdf available at https://authorea.com/users/406342/articles/517040-what-is-theimpact-of-a-previously-failed-robicsek-repair-in-the-subsequent-treatment-of-sternaldehiscence-with-thermoreactive-nitinol-clips

\section{Hosted file}

Table 2.pdf available at https://authorea.com/users/406342/articles/517040-what-is-theimpact-of-a-previously-failed-robicsek-repair-in-the-subsequent-treatment-of-sternaldehiscence-with-thermoreactive-nitinol-clips

\section{Hosted file}

Table 3.pdf available at https://authorea.com/users/406342/articles/517040-what-is-theimpact-of-a-previously-failed-robicsek-repair-in-the-subsequent-treatment-of-sternaldehiscence-with-thermoreactive-nitinol-clips 